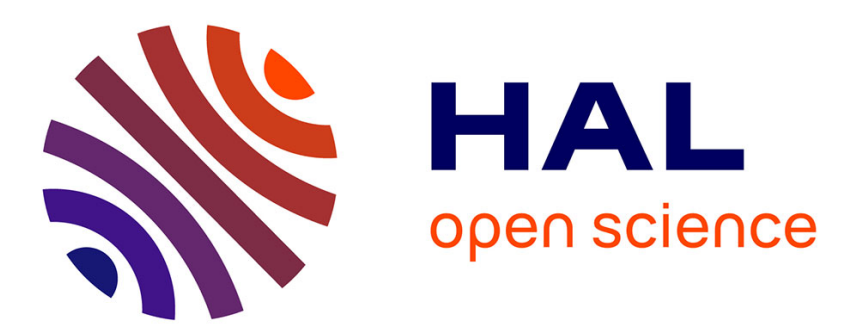

\title{
Resonant coupling between surface and interface plasma waves in high-density sharp-edged plasmas produced by ultrafast laser pulses
}

J. Kupersztych, Michèle Raynaud

\section{- To cite this version:}

J. Kupersztych, Michèle Raynaud. Resonant coupling between surface and interface plasma waves in high-density sharp-edged plasmas produced by ultrafast laser pulses. Physical Review E , 1999, 10.1103/PhysRevE.59.4559 . hal-01949718

\section{HAL Id: hal-01949718 \\ https://hal.science/hal-01949718}

Submitted on 26 Dec 2020

HAL is a multi-disciplinary open access archive for the deposit and dissemination of scientific research documents, whether they are published or not. The documents may come from teaching and research institutions in France or abroad, or from public or private research centers.
L'archive ouverte pluridisciplinaire HAL, est destinée au dépôt et à la diffusion de documents scientifiques de niveau recherche, publiés ou non, émanant des établissements d'enseignement et de recherche français ou étrangers, des laboratoires publics ou privés. 


\title{
Resonant coupling between surface and interface plasma waves in high-density sharp-edged plasmas produced by ultrafast laser pulses
}

\author{
J. Kupersztych and M. Raynaud \\ Commissariat à l'Energie Atomique, DRECAM/SRSIM, Bâtiment 462, Centre d'Etudes de Saclay, 91191 Gif-sur-Yvette Cedex, France
}

(Received 15 June 1998; revised manuscript received 14 December 1998)

\begin{abstract}
We consider the interaction between an ultrashort laser pulse and a hot high-density sharp-edged lasercreated plasma resulting from a microstructured target with a double-step density profile. We demonstrate that an electron plasma wave can be resonantly driven at the density jump between the two plasmas by the field of a laser-excited surface wave. Two different excitation regimes can exist depending on the wavelength and the angle of incidence of the laser. This effect may have interesting experimental consequences in hot electron generation and x-ray emission due to breaking of the resonant plasma wave. [S1063-651X(99)06904-4]
\end{abstract}

PACS number(s): 52.40.Nk, 52.50.Jm

\section{INTRODUCTION}

During the past decade, with the development of intense laser sources of ultrashort (femtosecond) pulse duration, laser-plasma interaction experiments have been able to study a new kind of physical situations that can only exist on such ultrashort time scales: laser-produced hot high-density plasmas, characterized by very steep density gradients $[1,2]$, where the hydrodynamic expansion does not play a dominant role. Much attention has been drawn to possible applications of these unusual plasmas to generate ultrafast pulses of $\mathrm{x}$ radiation in the $\mathrm{keV}$ range [2].

Actually, for sharp-edged overdense plasmas, some basic absorption mechanisms such as resonant absorption and parametric instabilities $[3,4]$ cannot occur with efficiency and other specific absorption mechanisms such as "vacuum heating" [5], anomalous skin effect [6], and sheath inverse bremsstrahlung [7] have been alternatively put forward. Now, reflectivity measurements have indicated [1] that absorption of the laser energy mainly occurs at the surface of the solid before it expands. On the other hand, emission of $\mathrm{x}$ rays, which is a valuable experimental signature of energy absorption by laser-produced plasmas, has been recently shown to be enhanced by using a variety of microstructured targets such as gratings and porous forms of metals [8], which seems to indicate the importance of the target structure for laser absorption in the femtosecond regime.

In fact, the existence of structured surfaces allows the coupling between a laser field and a particular form of electron collective oscillations that specifically depend on the density jump at the surface, namely, the so-called surface plasma waves [9-11]. In the present study, we wish to take into account this peculiarity by considering a different absorption mechanism that may exist when the density profile has the form of a double step, composed of a plasma of electron number density $n_{0}^{(I)}$ overlaid by another homogeneous plasma of thickness $h$ and of electron number density $n_{0}^{(I I)}$, with $n_{0}^{(I I)}<n_{0}^{(I)}$ (see Fig. 1). Our idea is to create the conditions under which a resonant electron plasma wave may be driven at the density jump located at the interface between the two plasmas, by the field of a surface wave which would be conveniently excited on the target surface.
This could be done, for example, by using a grating coupler [9] which is a device that allows momentum conservation between the photon momentum $\omega / c$ and the surface wave vector $\mathbf{k}$ to be satisfied. The frequency $\omega$ and wave vector $\mathbf{k}$ of the resonantly driven plasma wave will be therefore such that $\quad \omega<\omega_{p}^{(I I)}<\omega_{p}^{(I)}$, with $\omega / c \leqslant k$, where $\omega_{p}^{(i)}$ $=\left(4 \pi e^{2} n_{0}^{(i)} / m\right)^{1 / 2}$ denotes the electron plasma frequency in each region of space $[i=I, i=I I$, and $i=v$ (vacuum) $]$. This condition will reflect a physical situation basically different from the known case $\omega_{p}^{(I I)}<\omega<\omega_{p}^{(I)}[10,11]$, inasmuch as the existence of the interfacial resonance will necessarily depend on the thickness of the plasma overlayer.

Actually, the characteristics of a surface plasma wave are closely analogous to the driver field created at the reflection point in a resonant absorption scheme as it is a highfrequency field, evanescent toward the plasma and decreasing slowly in the vacuum [11]. From this analogy, it may be inferred that a resonant-absorption-like mechanism can be created in a double-step density profile: the field of the surface wave can play the same role as the driver field created at the reflection point in a resonant absorption scheme and may excite a plasma resonance at the density jump between the two plasmas at the same frequency as that of the driving field of the surface wave. Hence, excitation of an interface plasma wave may represent an additional absorption mechanism

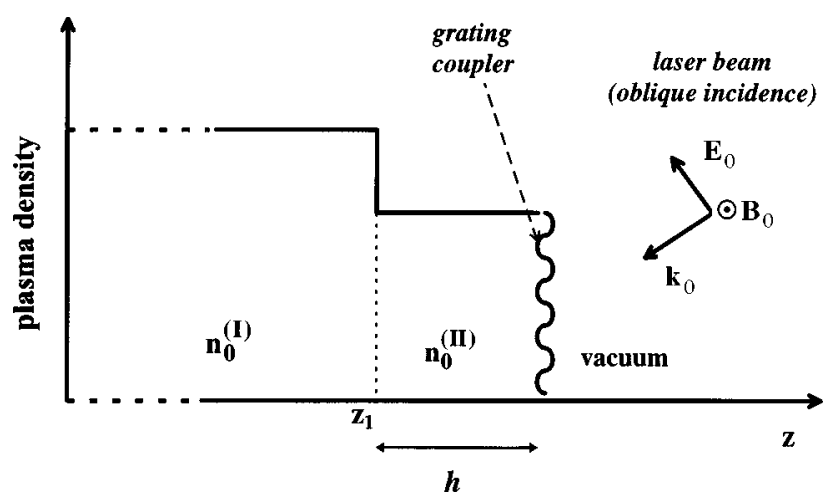

FIG. 1. Representation of the plasma density with respect to the coordinate $z$ normal to the semi-infinite plane of the target. The laser beam is impinging, in oblique incidence and $p$ polarization, onto the grating at the surface of the lower-density plasma. 
with respect to the absorption resulting solely from excitation of surface waves.

\section{RESONANT EXCITATION OF INTERFACE PLASMA WAVES}

For the sake of simplicity, we shall use a standard collisionless hydrodynamical approach [12]: the collective oscillations of the electron gas are described by the system of Maxwell equations coupled to the equation of continuity and the high-frequency fluid equation of motion, which, when linearized with respect to the electron density fluctuations $\widetilde{n}(\mathbf{r}, t)=n_{e}(\mathbf{r}, t)-n_{0}$, can be written as

$$
\partial \mathbf{v} / \partial t=-(e / m) \mathbf{E}-3 k_{B} T \nabla \tilde{n} / m n_{0},
$$

where $\mathbf{E}$ is the self-consistent electric field, $\mathbf{v}$ the fluid velocity, $T$ the electron temperature, and the last term represents the gradient of the kinetic pressure. It has been assumed that the temperature is only slowly varying in the plasma overlayer and near the interface between the two plasmas so that the temperature gradient contributes to a second-order term in the linearized high-frequency equation. Considering the time dependence in the form $e^{-i \omega t}$ and after taking the divergence of Eq. (1) and making use of the Poisson equation together with the linearized continuity equation, we immediately obtain

$$
\left(\omega^{2}-\omega_{p}^{(i) 2}+3 V_{\mathrm{th}}^{2} \Delta\right) \boldsymbol{\nabla} \cdot \mathbf{E}^{(i)}=0,
$$

where $V_{\mathrm{th}}=\left(k_{B} T / m\right)^{1 / 2}$ is the thermal velocity. By similar trivial manipulations, we can obtain the following equation for the rotational part of the electric field:

$$
\left(\omega^{2}-\omega_{p}^{(i) 2}+c^{2} \Delta\right) \nabla \times \mathbf{E}^{(i)}=\mathbf{0} .
$$

Equations (2a) and (2b) are the basic equations pertaining to our problem. The solution we are looking for is an electromagnetic wave of frequency $\omega$ and wave vector $\mathbf{k}$ directed along the $x$ axis: $\mathbf{E}=\left(E_{x}(z), 0, E_{z}(z)\right) e^{-i \omega t+i k x}$, traveling along each interface (plasma $I$-plasma $I I$ at $z=z_{1}$ and plasma II-vacuum at $z=z_{2}=z_{1}+h$ ) and decaying toward the plasma along the $z$ axis. In this way, Eq. (2a) and (2b) take the simpler form:

$$
\begin{aligned}
& \left(\partial^{2} / \partial z^{2}-\gamma^{(i) 2}\right)\left(k E_{x}^{(i)}-i \partial E_{z}^{(i)} / \partial z\right)=0, \\
& \left(\partial^{2} / \partial z^{2}-\alpha^{(i) 2}\right)\left(k E_{z}^{(i)}-i \partial E_{x}^{(i)} / \partial z\right)=0,
\end{aligned}
$$

where the coefficients $\gamma^{(i)}$ and $\alpha^{(i)}$ are defined as $\gamma^{(i) 2}=k^{2}$ $+\left(\omega_{p}^{(i) 2}-\omega^{2}\right) / 3 V_{\mathrm{th}}^{2}$ and $\alpha^{(i) 2}=k^{2}+\left(\omega_{p}^{(i) 2}-\omega^{2}\right) / c^{2}$. The particular solution corresponding to the relevant case $\omega<\omega_{p}^{(I I)}$ $<\omega_{p}^{(I)}$, together with the condition $\omega / c \leqslant k$, requires the coefficients $\gamma^{(i)}$ and $\alpha^{(i)}$ to be real. If we take $\gamma^{(i)}>0$ and $\alpha^{(i)}>0$, the sought-after solution can therefore be written in each region of the plasma and in vacuum in the following form:

$$
\begin{aligned}
E_{x}^{(i)}(z)= & k\left(C_{(i)} e^{\gamma^{(i)} z}+C_{(i)}^{\prime} e^{-\gamma^{(i)} z}\right) \\
& +i \alpha^{(i)}\left(D_{(i)} e^{\alpha^{(i)} z}-D_{(i)}^{\prime} e^{-\alpha^{(i)} z}\right),
\end{aligned}
$$

$$
\begin{aligned}
E_{z}^{(i)}(z)= & i \gamma^{(i)}\left(-C_{(i)} e^{\gamma^{(i)} z}+C_{(i)}^{\prime} e^{-\gamma^{(i)} z}\right) \\
& +k\left(D_{(i)} e^{\alpha^{(i)} z}+D_{(i)}^{\prime} e^{-\alpha^{(i)} z}\right) .
\end{aligned}
$$

In the above expressions the coefficients $C_{(i)}, D_{(i)}, C_{(i)}^{\prime}$, and $D_{(i)}^{\prime}$ are then determined by taking into account the requirement of no divergence of the field when $z \rightarrow \pm \infty$ and by using the boundary conditions of the problem, to wit: continuity of all the components of the electric field at $z=z_{1}$ and $z=z_{2}$ together with continuity of all the components of the (linearized) electron current density $\mathbf{j}(\mathbf{r}, t)=-e n_{0} \mathbf{v}(\mathbf{r}, t)$ at $z=z_{1}$, and continuity of the normal component $j_{z}$ at $z=z_{2}$ (along with the assumptions $E_{y}=0$ and $j_{y}=0$ ). After some tedious but trivial algebra, we obtain the sought-after dispersion relation $\omega(k)=0$, which is nothing more than the determinantal equation of the linear equation system between the above coefficients:

$$
\begin{aligned}
& \left(\gamma^{(I)}-\gamma^{(I I)}\right)\left(S_{1}^{-}+S_{2}^{-}\right)+\left(\gamma^{(I)}+\gamma^{(I I)}\right)\left(S_{1}^{+}+S_{2}^{+}\right) \\
& +2 \gamma^{(I)} \Omega-2 k(1+\mu)(1-\eta)=0,
\end{aligned}
$$

where we have noted

$$
\begin{gathered}
\mu=\left(\alpha^{(I I)}-\alpha^{(I)}\right) /\left(\alpha^{(I I)}+\alpha^{(I)}\right), \\
\eta=\left(\omega_{p}^{(I I) 2}-\omega^{2}\right) /\left(\omega_{p}^{(I) 2}-\omega^{2}\right), \\
\Omega=(1-\mu)\left(\alpha^{(I I)} / k\right)-\eta(1+\mu)\left(\alpha^{(I)} / k\right),
\end{gathered}
$$

and

$$
\begin{aligned}
S_{1}^{ \pm}= & {\left[\left(\alpha^{(v)} / k \pm k / \gamma^{(I I)}\right)\left(\omega_{p}^{(I I)} / \omega\right)^{2}\right.} \\
& \left.-\left(\alpha^{(v)}+\alpha^{(I I)}\right) / k\right] e^{\left( \pm \gamma^{(I I)}+\alpha^{(I I)}\right) h,} \\
S_{2}^{ \pm}= & {\left[\left(\alpha^{(v)} / k \pm k / \gamma^{(I I)}\right)\left(\omega_{p}^{(I I)} / \omega\right)^{2}\right.} \\
& \left.-\left(\alpha^{(v)}-\alpha^{(I I)}\right) / k\right] \mu e^{\left( \pm \gamma^{(I I)}-\alpha^{(I I)}\right) h} .
\end{aligned}
$$

Furthermore, the determination of the coefficients enables us to obtain the explicit expressions for the components of the self-consistent electric field in each region of the plasma and in vacuum as a function of an unique arbitrary coefficient $G$. This coefficient can be related to the amplitude of the external electromagnetic wave (in the same way as the amplitude of the driver field in resonant absorption is proportional to the amplitude of the external $e-m$ wave [13]). Therefore, in region I of the plasma the components of the field are

$$
\begin{aligned}
E_{z}^{(I)}(z)= & \left\{\left\{\left[\left(S_{1}^{-}+S_{1}^{+}+S_{2}^{-}+S_{2}^{+}\right)+2 \Omega\right] \gamma^{(I)} e^{\gamma^{(I)}\left(z-z_{1}\right)}\right.\right. \\
+ & \left.2 k(1+\mu) \eta e^{\alpha^{(I)}\left(z-z_{1}\right)}\right\} e^{\alpha^{(I I)} z_{1}} \\
E_{x}^{(I)}(z)= & 2 i G e^{\alpha^{(I I)}} z_{1}\left\{e ^ { \gamma ^ { ( I ) } ( z - z _ { 1 } ) } \left[(1-\mu) \alpha^{(I I)}\right.\right. \\
& \left.-(1+\mu) \eta \alpha^{(I)}+\frac{1}{2} k\left(S_{1}^{-}+S_{1}^{+}+S_{2}^{-}+S_{2}^{+}\right)\right] \\
& \left.+\eta \alpha^{(I)}(1+\mu) e^{\alpha^{(I)}\left(z-z_{1}\right)}\right\} .
\end{aligned}
$$

In region II, they are 


$$
\begin{aligned}
& E_{z}^{(I I)}(z)=G\left[\left(S_{1}^{-}+S_{2}^{-}\right) \gamma^{(I I)} e^{\gamma^{(I I)}\left(z-z_{1}\right)}\right. \\
& -\left(S_{1}^{+}+S_{2}^{+}\right) \gamma^{(I I)} e^{-\gamma^{(I I)}\left(z-z_{1}\right)} \\
& \left.+2 k\left(e^{\alpha^{(I I)}\left(z-z_{1}\right)}+\mu e^{-\alpha^{(I I)}\left(z-z_{1}\right)}\right)\right] e^{\alpha^{(I I)} z_{1}}, \\
& E_{x}^{(I I)}(z)=i G e^{\alpha^{(I I)}} z_{1}\left\{2 \alpha^{(I I)}\left(e^{\alpha^{(I I)}\left(z-z_{1}\right)}-\mu e^{-\alpha^{(I I)}\left(z-z_{1}\right)}\right)\right. \\
& +k e^{\gamma^{(I I)}\left(z-z_{1}\right)}\left(S_{1}^{-}+S_{2}^{-}\right) \\
& \left.+k e^{-\gamma^{(I I)}\left(z-z_{1}\right)}\left(S_{1}^{+}+S_{2}^{+}\right)\right\}
\end{aligned}
$$

and in vacuum, they take the form

$$
\begin{aligned}
E_{z}^{(v)}(z)= & 2 k G\left(1-\omega_{p}^{(I I) 2} / \omega^{2}\right) \\
& \times\left(e^{\alpha^{(I I)} h}+\mu e^{-\alpha^{(I I)} h}\right) e^{\alpha^{(I I)} z_{1}} e^{-\alpha^{(v)}\left(z-z_{2}\right)}, \\
E_{x}^{(v)}(z)= & -2 i G \alpha^{(v)}\left(1-\omega_{p}^{(I I)^{2}} / \omega^{2}\right) \\
& \times e^{-\alpha^{(v)}\left(z-z_{2}\right)} e^{\alpha^{(I I)} z_{1}}\left[e^{\alpha^{(I I)} h}+\mu e^{-\alpha^{(I I)} h}\right] .
\end{aligned}
$$

Owing to the complexity of the above formula, the influence of the relevant parameters (the overlayer thickness $h$ and the wave vector $\mathbf{k}$ ) is difficult to perceive, apart from some striking features. Thus, owing to the exponential dependence along the $z$ axis, the analytic expressions (4)-(6) exhibit clearly strong sharp maxima of the field amplitude at the point $z=z_{2}$, that is, at the interface with the vacuum, which is none other than the normal component of the surface plasma wave, and at the point $z=z_{1}$, which is none other than the resonant field of the plasma wave located at the interface between the two plasmas. As mentioned above, the excitation of the resonant interface plasma wave results from the evanescent field of the surface wave tunneling throughout the external region (II) of the plasma. Hence, it can be inferred that the magnitude of the effect should depend on both the thickness of the plasma overlayer and the amplitude of the wave vector.

The role of the overlayer thickness $h$ is already exhibited in expression (5), by factors of the type $\exp \left[-\left(\alpha^{(I I)}\right.\right.$ $\left.\left.+\gamma^{(I I)}\right) h\right]$ and $\exp \left[\left(\alpha^{(I I)}-\gamma^{(I I)}\right) h\right]\left(\right.$ with $\left.\alpha^{(I I)}-\gamma^{(I I)}<0\right)$, which results in a decreasing of the resonance amplitude as the thickness increases [note that the term $S_{1}^{+}+S_{2}^{+} \rightarrow 0$ when $h$ $\rightarrow \infty$ because of the dispersion relation (3)]. Hence, the resonant interface plasma wave disappears for too large a thickness, in keeping with the intuitive interpretation of the phenomenon and with the above analogy with the resonantabsorption process.

The role of the wave vector is somewhat different. In the present study, we seek a coupling between a surface wave of phase velocity $\omega / k$ and density fluctuations at the interface between the two plasmas. As the collective interactions, due to the long-range Coulomb forces, travel with the light velocity, the coupling between the driving field of the surface wave and the excited wave at the plasma (I)-plasma (II) interface will therefore depend on the value of $\omega / k$ with respect to $c$. Thus, the efficiency of the coupling will be, respectively, optimized or restrained depending on whether the phase velocity $\omega / k$ is close to or much smaller than the velocity of light.

\section{DISCUSSION OF THE RESULTS}

In order to explore the influence of the relevant parameters, we have studied numerically the example of a target composed of gold covered with an aluminium layer of thickness $h=100-200 \AA$. This target is assumed to be illuminated by a strong laser pulse with an intensity of the order of $10^{15} \mathrm{~W} / \mathrm{cm}^{2}$. An average electron energy ("temperature") of $300 \mathrm{eV}$ corresponding to experimental measurements (100 $\mathrm{eV}-1 \mathrm{keV})[1,2]$ has been assumed. The resulting plasma densities may be crudely estimated by means of the following ultrasimplified arguments. At such laser intensities, ionization of the metals is expected to provide high- $Z$ ions having quasiclosed shells. Hence, gold is expected to leads to nickel-like ions $(Z=51)$ and aluminium to heliumlike ions $(Z=11)$. Furthermore, due to expansion of the plasma, the ion densities $n_{i}$ may be estimated to correspond to the metal densities divided by a factor of 10 for aluminium and a factor of 2 in the case of gold. Hence, the plasma electron densities $n_{e}=Z n_{i}$ may be crudely estimated to be $n_{e}=1.5$ $\times 10^{24} \mathrm{~cm}^{-3}$ for the gold plasma and $n_{e}=6.6 \times 10^{22} \mathrm{~cm}^{-3}$ for the aluminium overlayer plasma.

As the order of magnitude of a surface-wave frequency is roughly $\omega \approx \omega_{p} / \sqrt{2}$ (for small values of $k$ ) and since $\left(\omega_{p}^{(\mathrm{Au})} / \sqrt{2}\right) / c \approx 3 \times 10^{5} \mathrm{~cm}^{-1}$, two (realistic) situations will therefore deserve to be examined: $k \approx 10^{5}$ and $10^{6} \mathrm{~cm}^{-1}$. In Figs. 2 and 3 are reported the amplitude variations of the normal component of the self-consistent electric field $E_{z}(\omega, k)$ with respect to the $z$ coordinate, where the coefficient $G$, which is proportional to the amplitude of the external laser field (in vacuum), has been taken to be unity. Obviously, the frequency $\omega$ is, for each overlayer thickness $h$ $=100$ and $200 \AA$, given by the solution of the dispersion relation (3) (represented in Fig. 4 for the case $h=100 \AA$ ) as a function of the wave vector $\mathbf{k}$.

In Fig. 2(a), the amplitude of the electric field exhibits a strong enhancement at the plasma I-plasma II interface (at $z=-h=-100 \AA$ ) in comparison with the amplitude of the surface wave (at $z=0$ ). The important part played by the overlayer thickness, which is expected as a consequence of a tunnel effect, appears clearly on Figs. 2(a) and 3(a), 2(b) and 3(b). Thus, when the thickness of the overlayer increases from $h=100-200 \AA$, the resonance amplitude decreases by nearly a factor of 2 .

As shown in Figs. 3(a) and 3(b), the role of the wave vector is also particularly important in this problem for laser energy absorption inasmuch as two different regimes seem to exist depending on the value of $k$. Thus, for large values of $k$ $\left(k \approx 10^{6} \mathrm{~cm}^{-1}\right)$, the amplitude of the resonant plasma wave at the interface between the two plasmas becomes of the same order as that of the driving surface wave while the field diffuses throughout the bulk of the overlayer instead of being localized at the interface. This case will likely correspond to a thermal-like absorption regime where the plasma resonance, spreaded in the whole plasma overlayer, will be rather dissipated by electron-electron collisions, thus increasing thermal heating of the overlayer. Obviously, the temperature will subsequently evolve during a longer time scale because of thermal conduction, electron-ion energy transfer and hydrodynamic expansion into the vacuum.

In the case of small values of $k\left(k \approx 10^{5} \mathrm{~cm}^{-1}\right)$ where the 

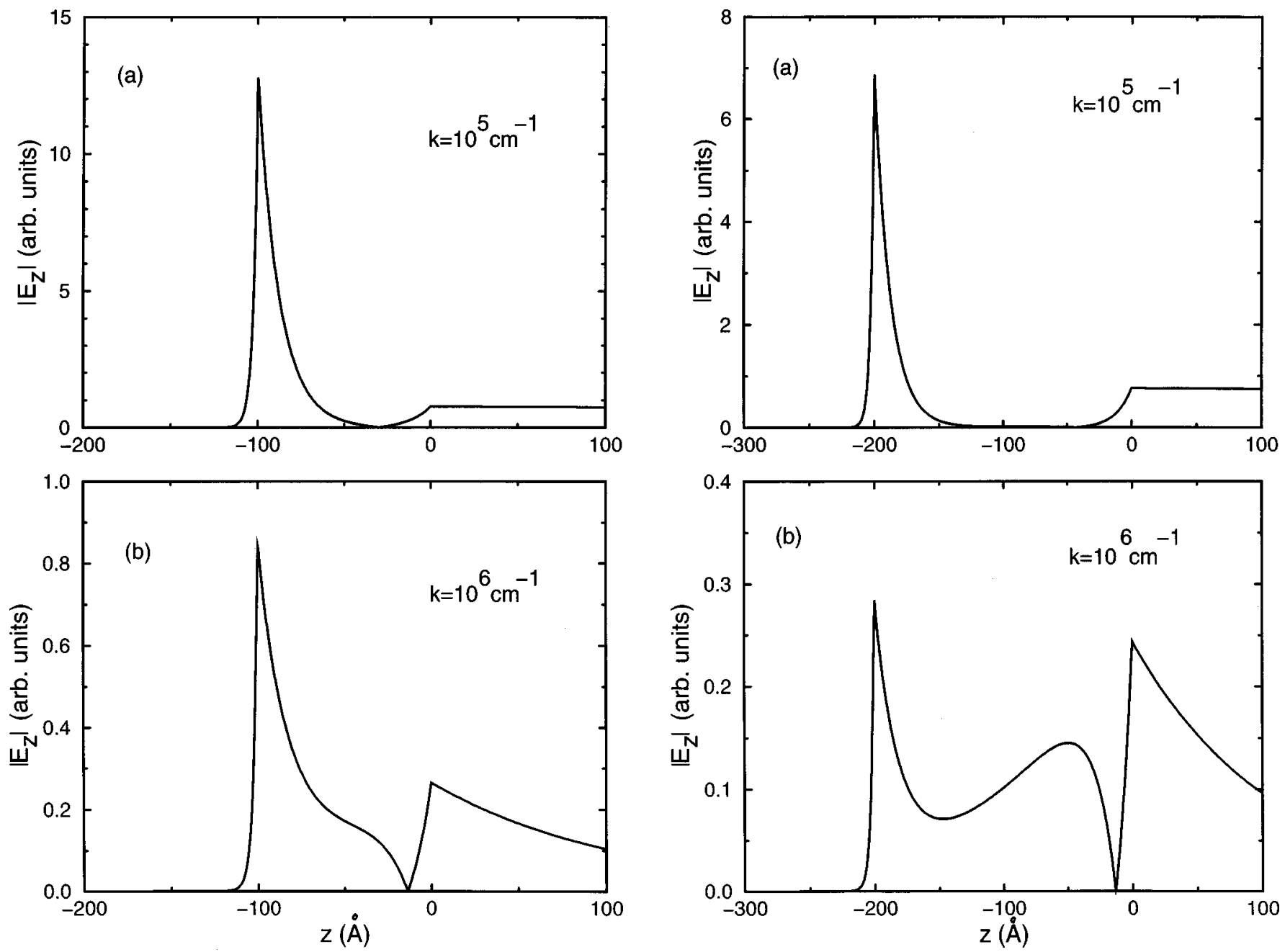

FIG. 2. Representations of the normal component of the selfconsistent electric field $E_{z}(\omega, k)$ versus normal coordinate $z$. The thickness of the overlayer is $h=100 \AA$. The ratio $R$ between the amplitudes of the interface and surface waves are $R=18$ for case (a) and $R=3.2$ for (b).

resonance is well localized at the interface between the two plasmas and of strong amplitude, dissipation of the resonant wave energy will likely occur via nonlinear processes such as breaking of the resonant plasma wave [4], leading to generation of energetic electrons and, consequently, to emission of $x$ radiation via bremsstrahlung. In fact, it is generally admitted from solid-state studies [9-14] that the amplitude of the surface plasmon field resonantly excited by a laser beam is at least of the order of 10 times the amplitude of the laser field $E_{L}$. In other words, the coefficient $G$ may be estimated of the order of $10 E_{L}$. Now, for the case considered in Fig. 2(a), the order of magnitude of the interface plasma wave field $E$ is nearly $10 G$, that is (at least), $10^{2} E_{L}$. As the laser pulse intensity considered here is $I_{L} \approx 10^{15} \mathrm{~W} / \mathrm{cm}^{2}, E_{L}$ is of the order of $10^{6} \mathrm{statvolt} / \mathrm{cm}$, and the amplitude of the interface plasma wave field is therefore of the order of $10^{8} \mathrm{statvolt} / \mathrm{cm}$. It follows that the quiver velocity $v_{\text {osc }}$ $=e E / m \omega$ of an electron in the field of the interface plasma wave is in the relativistic domain. Now, the well-known onedimensional cold-plasma condition for breaking of an intense plasma wave is [15] $e E / m \omega \approx \omega / k$. Actually, for a hot plasma such as that considered in the present discussion, the

FIG. 3. Same caption as for Fig. 2 but the thickness of the overlayer here is $h=200 \AA$. The amplitude ratios are $R=9$ for (a) and $R=1.2$ for (b).

field amplitude of the plasma wave required for wave breaking is even lower [16]. Hence, relativistic breaking of the resonant interface plasma wave considered here is expected to occur, as can be shown explicitly in the following example.

Consider laser-target coupling by means of a grating with groove spacing $a \approx 2 \mu \mathrm{m}$. As we know, the laser frequency $\omega$, the angle of incidence $\theta$, and the wave vector $\mathbf{k}$ of the excited plasma wave are related through the relation $k$ $=(\omega / c) \sin \theta \pm 2 \pi / a$ (for the first harmonic) [9], which results from momentum conservation. For $k \approx 10^{5} \mathrm{~cm}^{-1}$ and $\theta$ $\approx 60^{\circ}$ (typically), the frequency of the plasma wave is $\omega$ $=2.39 \times 10^{15} \mathrm{rad} / \mathrm{s}$. The required amplitude of the plasma wave for cold plasma wave breaking, $E=m \omega^{2} / k e=1.1$ $\times 10^{8}$ statvolt $/ \mathrm{cm}$, is then satisfied for the above value of the interface plasma wave field. Actually, as we deal with a hot plasma, the wave-breaking condition will be fully fulfilled. Thus, wave breaking appears as an important mechanism for dissipation of the resonant interface plasma wave and consequently, for laser energy absorption in the long-wavelength regime. Now, these two frequency-dependent absorption regimes [namely, short (thermal) and long (hot-electron generation) wavelength regimes) are features that are also characteristic of resonant absorption itself. It is therefore 


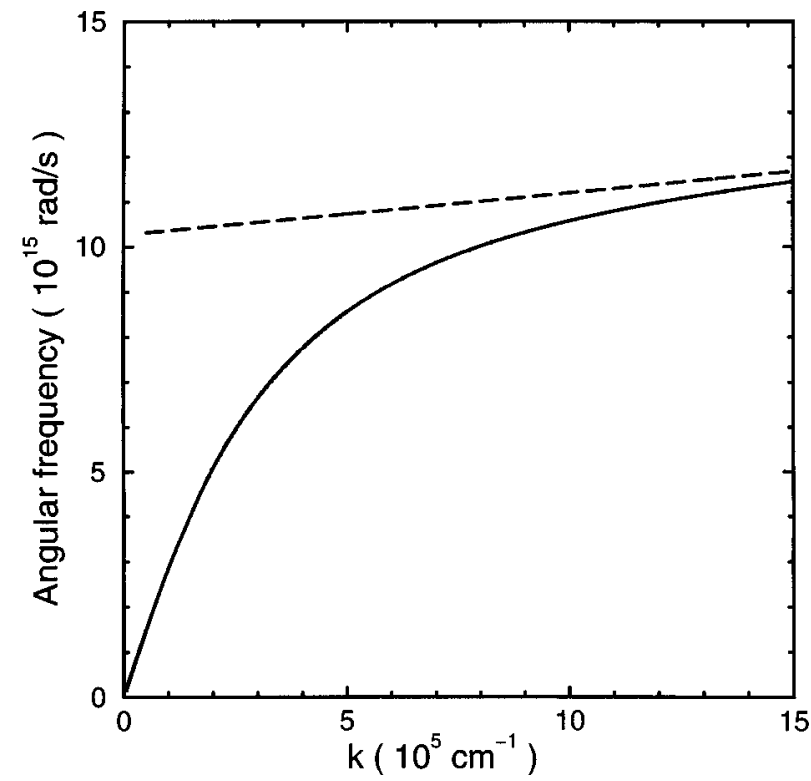

FIG. 4. Representation of the dispersion relation $\omega(k)=0$ given by Eq. (3). The dotted line represents the asymptotic limit $\omega(k)$ for large values of $k$.

necessary to differentiate the relative contributions of these two mechanisms.

The essential differences between resonant absorption (resonant excitation of volume plasma waves at critical density) and resonant excitation of surface and interface plasma waves by means of a grating coupler, lie in the values of the electron density gradient and the angle of incidence of the laser beam. In the case of a target with a rippled surface, resonant absorption may occur for a rather large range of incidence angles (even including normal incidence), a fact that would not be true, of course, for a flat surface. On the contrary, resonant excitation of surface (and interface) plasma waves needs conservation of energy and momentum as expressed by the relation $k=(\omega / c) \sin \theta \pm 2 \pi / a$, which requires a definite value of the incidence angle $\theta$ (for given laser frequency $\omega$ and groove spacing $a$ of the grating) of the laser beam. Furthermore, the shape of the electron density profile plays an essential part both for surface wave excitation and resonant absorption. Resonant excitation of surface waves and, consequently, excitation of interface plasma waves, are optimized by stepwise density gradients. On the other hand, resonant absorption is nearly nonexistent for steep density gradients. Hence, resonant absorption and excitation of surface and interface plasma waves at the corresponding resonance frequency can be seen as two complementary absorption mechanisms that may occur at different stages of the laser-plasma interaction in the femtosecond regime. Thus, in the early stages of the interaction (a few tens of femtoseconds) where the plasma density profile is still similar to that of the solid target, resonant excitation of the interface plasma waves discussed here may represent a significant absorption process. For longer time scales, the hydrodynamical expansion of the plasma will lead to an increase in the electron density gradient length and resonant absorption will then take place. Other mechanisms, such as sheath formation [17] at the surface and the interface, will then also appear and contribute to laser energy absorption. It can therefore be concluded that the use of double-layer microstructured targets may yield additional energy absorption of ultrafast laser pulses due to resonant excitation of interface plasma waves and, in the case of short laser wavelengths, to additional electron generation of hot electrons together with $\mathrm{x}$-radiation emission via bremsstrahlung of the high-energy electrons.

\section{ACKNOWLEDGMENTS}

The authors acknowledge N. Auby for her help with the numerical computations and Dr. C. Chenais-Popovics for fruitful discussions.
[1] H. M. Milchberg, R. R. Freeman, and S. C. Davey, Phys. Rev. Lett. 61, 2364 (1988); M. M. Murnane, H. C. Kapteyn, and R. W. Falcone, ibid. 62, 155 (1989); H. M. Milchberg and R. R. Freeman, Phys. Fluids B 2, 1395 (1990).

[2] J. A. Cobble, G. A. Kyrala, A. A. Hauer, A. J. Taylor, C. C. Gomez, N. D. Delamater, and G. T. Schappert, Phys. Rev. A 39, 454 (1989); J. A. Cobble, G. T. Schappert, L. A. Jones, A. J. Taylor, G. A. Kyrala, and R. D. Fulton, J. Appl. Phys. 69, 3369 (1991); J. C. Gauthier, J. P. Geindre, P. Audebert, S. Bastiani, C. Quoix, G. Grillon, A. Mysyrowicz, A. Antonetti, and R. C. Mancini, Phys. Plasmas 4, 1811 (1997).

[3] D. W. Forslund, J. M. Kindel, L. Kenneth, E. L. Lindman, and R. L. Morse, Phys. Rev. A 11, 679 (1975); K. G. Estabrook, E. J. Valeo, and W. L. Kruer, Phys. Fluids 18, 1151 (1975).

[4] J. Albritton and P. Koch, Phys. Fluids 18, 1136 (1975).

[5] F. Brunel, Phys. Rev. Lett. 59, 52 (1987); F. Brunel, Phys. Fluids 31, 2714 (1988).

[6] E. M. Lifshitz and L. P. Pitaevskii, Physical Kinetics (Pergamon, Oxford, 1981), p. 368; W. Rosmus and V. T. Tikhon- chuk, Phys. Rev. A 42, 7401 (1990); J. P. Matte and K. Aguenaou, ibid. 45, 2558 (1992).

[7] P. J. Catto and R. M. More, Phys. Fluids 20, 704 (1977); T.-Y. Brian Yang, William L. Kruer, Richard M. More, and A. Bruce Langdon, Phys. Plasmas 2, 3146 (1995).

[8] S. P. Gordon, T. Donnelly, A. Sullivan, H. Hamster, and R. W. Falcone, Opt. Lett. 19, 484 (1994).

[9] H. Raether, Surface Plasmons on Smooth and Rough Surfaces and on Gratings (Springer, Berlin, 1988), pp. 8 and 9.

[10] P. K. Kaw and J. B. Mac Bride, Phys. Fluids 13, 1784 (1970); J. M. Kindel, K. Lee, and E. L. Lindman, Phys. Rev. Lett. 34, 134 (1975); H. Maki and K. Niu, J. Phys. Soc. Jpn. 45, 269 (1978); R. Dragila and E. G. Gamaliy, Phys. Rev. A 44, 6828 (1991).

[11] C. S. Liu and V. K. Tripathi, Interaction of Electromagnetic Waves with Electron Beams and Plasmas (World Scientific, Singapore, 1994), p. 32.

[12] See, e.g., W. L. Kruer, The Physics of Laser Plasma Interactions (Addison-Wesley, Redwood City, 1988), Chaps. 1.4 and 1.5 . 
[13] See, e.g., C. S. Liu and V. K. Tripathi, Interaction of Electromagnetic Waves with Electron Beams and Plasmas (Ref. [11]), Chap. 3.

[14] F. J. Garcia-Vidal and J. B. Pendry, Phys. Rev. Lett. 77, 1163 (1996); M. B. Sobnack, W. C. Tan, N. P. Wanstall, T. W. Preist, and J. R. Sambles, ibid. 80, 5667 (1998).
[15] J. Dawson, Phys. Rev. 113, 383 (1959).

[16] T. P. Coffey, Phys. Fluids 14, 1402 (1971); Z. M. Sheng and J. Meyer-ter-Vehn, Phys. Plasmas 4, 493 (1997).

[17] S. Eliezer and H. Hora, IEEE Trans. Plasma Sci. 17, 290 (1989). 